\title{
MOVEMENT DISORDERS IN CHILDREN
}

Sujitnath Choudhury1

${ }^{1}$ Associate Professor, Department of Paediatrics, Teacher of Government Medical College of Assam, Recognised by IMC.

ABSTRACT

Involuntary movements of different types are associated with many diseases in children. Movement disorders in adult have been published in different journals, but in children these disorders have been ignored, even in most of the paediatric neurology books. Here is a brief attempt to describe different types of movement disorders and their various names in different diseases. Possible investigations and treatment of the disorders have been described in short.

\section{KEYWORDS}

Movement Disorders, Dystonea.

HOW TO CITE THIS ARTICLE: Choudhury S. Movement disorders in children. J. Evolution Med. Dent. Sci. 2016;5(7):340-342,

DOI: $10.14260 /$ jemds/2016/74

\section{INTRODUCTION}

Most of us can control our body movement, but many children and adult cannot control it and even impossible for them. Movement disorders may have excess movement and those having diminished movement.

\section{HYPOKINESIAS}

- Slowness of movement is called bradykinesia

- Slowness to initiate movement is called akinesia and rigidity.

\section{HYPERKINESIAS}

- Tremor.

- Chorea

- Athetosis.

- Myoclonus.

- Ballism.

- Tics.

- Compulsion.

- Stereotypies movement.

- Mannerism.

- Akathisia.

- Restless leg syndrome.

Causes of Movement Disorders in Children: (I) Onset below 3 Years of Age

- Kernicterus.

- Perinatal asphyxia.

- Physiological chorea in infancy.

- Post cardio-pulmonary bypass.

(II) Onset after 3 Years of Age: Immunological

- Sydenham's chorea.

- SLE.

\section{INFECTION}

- Viral encephalitis.

- ADEM.

Financial or Other, Competing Interest: None.

Submission 14-12-2015, Peer Review 25-12-2015,

Acceptance 01-01-2016, Published 25-01-2016.

Corresponding Author:

Dr. Sujitnath Choudhury,

Door No. 14, Silchar Medical College,

Silchar, Assam.

E-mail: sujitnath_2002@rediffmail.com

DOI: $10.14260 /$ jemds $/ 2016 / 74$

\section{DRUG INDUCED}

- Neuroleptic.

\section{METABOLIC}

- Hepatic encephalopathy.

- Uremia.

- Hypoparathyroidism.

- PEM.

- Hyponatremia.

- Hypernatremia.

\section{GENETIC}

- Huntington's disease.

- Primary dystonea.

- Paroxysmal dystonea.

- Wilson's disease.

- Neuroacanthocytosis.

- Hallervorden-Spatz disease.

- Organic academia.

- GA, GM2 gangliosidosis.

\section{VASCULAR}

- Homocystinuria.

- Antiphospholipid antibody syndrome.

\section{(III) ONSET IN ADOLESCENCE}

- Wilson's disease.

- Hallervorden-Spatz disease.

- Juvenile Parkinsonism.

- Toxic

- Metabolic.

- Infectious.

- Immunological.

- Traumatic.

Common Causes of Movement Disorders in Children. ${ }^{1}$ Sydenham's Chorea

Chorea is the late manifestations of rheumatic fever or it may manifest without any other features of rheumatic fever, which is called lone chorea. It may be confined to one half of body called hemichorea. Sydenham's chorea may last from one month to 2 years and usually occurs 2-7 months after streptococcal infection. Jerky movements of hands and feet are like dancing continuous brisk purposeless movements involving distal muscle and disappear during sleep. Muscular hypotonea is common. 
Pronator sign in palm and wrist and trumpet sign in protracted tongues is found in Sydenham's chorea. Sydenham's chorea is treated with Haloperidol or sodium valproate for 5 to 6 months.

\section{Other causes of chorea are. 1}

- Choreoathetoid type of cerebral palsy.

- Lupus erythematosus.

- Henoch-Schonlein purpura.

- Anticardiolipin antibody syndrome.

\section{Huntington's Chorea. ${ }^{2}$}

Autosomal dominant condition may manifest in teenagers. Bradykinesia, rigidity and seizures along with choreiform movement and CT scan of brain show caudate atrophy.

\section{Primary Dystonia}

Genetic dystonia and some adult onset, focal dystonias.

\begin{tabular}{|l|l|}
\hline DYT $_{1} A D$ & Primary torsion dystonia \\
$\mathrm{D}_{2} \mathrm{~T}_{2} \mathrm{AR}$ & Primary torsion dystonia \\
$\mathrm{D}_{\mathrm{Y}} \mathrm{T}_{3}$ & Filipino dystonia parkinsonism \\
$\mathrm{Dy}_{4}$ & Primary torsion dystonia with laryngeal \\
$\mathrm{D}_{\mathrm{y}} \mathrm{T}_{5}$ & involvement \\
$\mathrm{DyT}_{6}$ & Dopa responsive dystonia \\
$\mathrm{D}_{\mathrm{y}} \mathrm{T}_{7}$ & Mixed phenotype primary torsion dystonia \\
$\mathrm{D}_{\mathrm{y}} \mathrm{T}_{8}$ & Paroxysmal non-kinesigenic choreoathetosis. \\
$\mathrm{D}_{\mathrm{y}} \mathrm{T}_{9}$ & DyT8 spasticity \\
DyT$_{10}$ & Paroxysmal kinesigenic chorea \\
$\mathrm{D}_{\mathrm{y}} \mathrm{T}_{11}$ & Familial myoclonus dystonia \\
\hline
\end{tabular}

\section{DyT1 Dystonia}

Autosomal dominant condition and commonest cause of early onset generalized dystonia. Dystonia typically begins in one limb, leg or arm and subsequently become generalized. The dystonia may not improve with treatment and can be severe and disability. Anticholinergic Baclofen and surgical procedures are often helpful.

\section{DyT5 Dystonia}

This is Dopa-responsive dystonia with diurnal fluctuation childhood onset dystonia with a dramatic and sustained response to low dose of levodopa. This type of dystonia usually starts at 6 years of age, but onset may be from infancy to adult life. Dystonia symptoms are more severe later in the day and improved in the morning. This condition is also known as Segawa's syndrome and respond to low dose of Levodopa.

\section{DyT10 Paroxysmal Kinesigenic Choreoathetosis. ${ }^{1}$}

Commonest cause of paroxysmal dyskinesia. The symptoms usually begin in childhood or adolescence. The attacks are brief lasting less than five minutes and may occur several times a day; respond to anticonvulsant therapy.

\section{DyT8 and DyT9}

Autosomal dominant condition, presents with paroxysmal dystonia choreoathetosis. The attacks are precipitated by emotional stress, fatigue and may last for 2 minutes to four hours and may occur up to 20 times a day.

\section{HEREDODEGENERATIVE DYSTONIAS. 4}

\section{Wilson's Disease}

Autosomal recessive condition onset is between 3 years to 58 years of age. Wilson's diseases manifest with dystonia, chorea, tremor, rigidity, spasticity and ataxia. Wilson's diseases is diagnosed by demonstrating Kayser-Fleischer ring in the cornea, decreased serum cereloplasmin level in blood, elevated 24 hours urinary copper and increased hepatic copper contents.

Wilson's disease is treated with copper chelating agent D-penicillamine which is to be continued for life long. Zinc salts has been found to be equally effective with lesser side effects than D-penicillamine.

Huntington's disease is a degenerative disease with autosomal dominant mode of transmission and presents with chorea, dementia, CT, ALRI which demonstrate caudate atrophy.

Neuroacanthocytosis is a cause of chorea in children and can be diagnosed by a fresh peripheral blood smear examination showing acanthocytes in the smear.

\section{Tics Disorder. 4,5}

Intermittent, repeated, stereotyped movements or sounds. Tics may be simple such as cough, grunt, facial twitch, shoulder shrug or complex such as word phrase or stereotyped sequence of movements, Tics are often associated with compulsions:

- Commonest tic disorder is transient tic of childhood.

- De la Tourette syndrome: It is a chronic multiple tic disorder, starts between 5 and 10 years of age common in males.

Vocal tics are frequent (Grunting and barking noises and coprolalia (Swear words).

\section{A child may fulfil criteria:}

- Multiple motor tics (Any kind of movement of the limbs, trunk or face).

- At least one vocal tic involving any sounds.

- Symptoms begin before the age of 21 years.

- Wax and wane of symptoms for more than one year.

Most of the tic disorders are treated with Haloperidol Risperidone and Pimozide.

\section{COMPULSIONS}

Complex behaviours different from tics that movement may be repetitive and ritualistic. Movements are often not stereotyped and having distinct purpose of the movement.

\section{Example}

Multiple unlocking and relocking of a door due to fear of thieves. Repeated hand washing due to fear of germs.

A need for symmetrical movements or placement of objects.

- Counting behaviour.

- Fixed routines.

- Obsessive thoughts.

Compulsions are usually treated with SSRI.

\section{STEREOTYPES}

Repetitive, stereotyped, purposeless movements that may be made by normal children when bored or excited.

Stereotypies are often associated with ADHD, autism or mental retardation. These movements may be voluntarily suppressed or behavioural therapy may control it. 


\section{AKATHISIA}

Describe a sense of restlessness leading to move or walk. The feeling is uncomfortable and the movements are voluntary. The sensation may lead to pacing or rubbing the legs, face or scalp with hands. Akathisias are common during medications like haloperidol or pimozide.

\section{Restless Leg Syndrome}

Unusual sensations like paresthesias dysesthesias under the skin leads the child to move or rub the legs and responds to $\mathrm{L}$. dopa and dopamine agonists.

\section{Tremor}

Essential Tremor. 4

Autosomal dominant condition having family history, present at an early age in successive generation.

\section{Infantile Tremor Syndrome}

It occurs in children between 6 months to 2 years of age with severe malnutrition due to magnesium deficiency. The tremor is rapid rhythmic and coarse and responds to magnesium supplementation.

\section{Kwashi Shakes}

Coarse tremors, Parkinsonism, rigidity, bradykinesia and myoclonus in children with PEM. It generally occurs 6 to several weeks after starting high protein diet.

\section{Drug Induced Movement Disorders \\ DYSTONIA}

- Akathisia.

- Parkinsonism.

- Tics.

- Tremor.

- Neuroleptic malignant syndrome.

\section{Focal Movement Disorder}

- Blepharospasm.

- Hemifacial spasm.

- Torticollis.

- Laryngeal dystonia.

- Oromandibular dystonia.

\author{
Possible Investigations. ${ }^{2}$ \\ Wood \\ - PBS - for Acanthocytes. \\ - LIT serum copper, serum ceruloplasmin, ANA. \\ - Hexoseaminidase. \\ - Blood glucose. \\ - Serum lactate and pyruvate. \\ - HIV antibodies.
}

\section{Urine: 24 Hours Urine Copper}

Bone Marrow: For blue histiocytes.

- Skin fibroblast for lysosomal enzyme assay. Liver biopsy for copper content.

- Slit lamp examination of eyes for EF ring.

- CT scan of brain.

- MRI of brain.

\section{Treatment of Dystonias.3,4}

All patients with childhood dystonia should receive a trial of Levodopa/carbidopa - combination as it is effective in DyT5 dystonia. ${ }^{2}$ If the response to Levodopa is partial, a dopamine agonist like Ropinirole/Pergolide can be added.

Patients who do not respond to the dopaminergic are to be tried with anticholinergic agents like Trihexyphenidyl and Benztropine. ${ }^{5}$ Trihexyphenidyl is started with $1 \mathrm{mg} 2$ times a day and later $0.5 \mathrm{mg}$ twice daily. When anticholinergics are ineffective other drugs that can be tried are Baclofen, Clonazepam, Lithium and Tetrabenazine.

\section{BOTULINUM TOXINS INFECTION. ${ }^{6}$}

It is highly effective in focal dystonia like cervical dystonia and blepharospasm hemifacial spasm.

\section{Surgical Treatment. ${ }^{4}$}

Implantation of catheters and pumps of intrathecal Baclofen in very small doses.

\section{THALAMOTOMY}

Deep brain stimulation where electrodes capable of delivering a continuous high frequency electrical stimulation are implanted in the basal ganglia.

Thalamotomy and pallidotomy for dystonia are other surgical procedures.

\section{REFERENCES}

1. Houser M, Soland V, Bhatia KP, et al. Paroxysmal kinesigenic choreoathetosis. J Neurol 1999;246:120-126.

2. Folstain SE, Leigh JR, Parhad IR. The diagnosis of Huntington's disease. Neurology 1966;36:1279-1283.

3. Marsden CD, Fahn S. The paroxysmal dyskinesias movement disorders, 3 Oxford Butterworth-Heinemann 1994;3rd ed:310-45.

4. Marsden $\mathrm{CD}$, Fahn S. Classification and investigation of dystonia movements disorder, 2 London Butterworth 1987;332-358.

5. Fletcher NA, Harding AE. A genetic study of idiopathic torsion dystonia in the United Kingdom. Brain 1990;113:379-95.

6. Greene P, Kang U, Brin M, et al. Double blind controlled trial of Botulinum toxin injection in spasmodic torticollis. Neurology 1990;40(8):1213-1218. 